Microparticles (MPs) (small membrane vesicles) have been reported in SLE patients, being an important source of autoantigens and inflammatory mediators. Based on these considerations, our study aims to measure the serum levels of sST2 in SLE patients, examining their association with disease activity and steroid consumption. Additionally, we aim to propose that MPs are an important source of ST2.

Methods Forty-six SLE patients were evaluated for disease activity (determined by SLEDAI), sST2 were measured by sandwich ELISA in serum samples and compared with 10 age- and sex-matched healthy controls (HCs). MPs were isolated from plasma from 9 SLE patients and $9 \mathrm{HC}$, and we evaluated the ST2 content in these vesicles by western blot.

Results Serum sST2 level was significantly higher in active SLE patients compared with HCs $(\mathrm{p}<0.001)$, and in inactive patients compared with HCs $(\mathrm{P}<0.01)$. We demonstrated higher sST2 levels among SLE patients on steroid treatment, with MPs from SLE patients containing ST2.

Conclusions We found elevated serum sST2 level in SLE patients, being higher in active patients; therefore ST2 could be an activity SLE biomarker. Additionally, MPs from SLE patients contain ST2, thus MPs could be an important source of circulating ST2, transporting and transferring ST2 to different cells for intercellular communication, consequently contributing to SLE pathogenesis.

Financial support Funding FONDECYT 1170648 and Programa de Sostenibilidad Universidad de Antioquia.

\section{P13 ASSESSING THE ABILITY OF ANTI-C1Q ANTIBODY MEASUREMENT TO PREDICT A FLARE OF LUPUS NEPHRITIS}

Sameer Bahal, Dev Pyne, Ravindra Rajakariar, Myles Lewis, Angela Pakozdi, Andrea CoveSmith. Barts Lupus Centre, Barts NHS Trust, London, UK

\subsection{6/lupus-2020-eurolupus.62}

Background Patients with lupus nephritis (LN) are at risk of relapse and hence long term disease monitoring is required. Here we undertook a prospective follow up of patients after having Anti-C1q antibodies (C1Q Ab) measurement to determine whether the result predicted a flare of LN.

Methods SLE patients attending an Inner-City Lupus Center, were involved in the study. A point-in-time measurement of $\mathrm{C} 1 \mathrm{Q} \mathrm{Ab}$ was made using an ELISA kit (Orgentec Diagnostika $\mathrm{GmbH}$ ). Medical records of patients were reviewed over the following 1 year to identify LN flares. A renal flare was defined as a doubling of the protein creatinine ratio with a subsequent decision to escalate immunosuppressive therapy.

Results 116 lupus patients were included in the study. Of those, 52 had biopsy proven LN (45\%). Positive C1Q ab was more common in patients with a history of biopsy proven $\operatorname{LN}(n=17,32.7 \%)$ compared to those with non-renal SLE $(n=10,15.6 \%),(p=0.03)$. Renal flares tended to be more common in $\mathrm{C} 1 \mathrm{q}$ ab positive $\mathrm{LN}$ patients $(\mathrm{n}=4,26.7 \%)$ compared to those without $\mathrm{C} 1 \mathrm{q}$ ab $(\mathrm{n}=2, \quad 7.14 \%)$. $(\mathrm{p}=0.782)$. Of the 64 patients with non-renal SLE, $1(10 \%)$ $\mathrm{C} 1 \mathrm{Q} \mathrm{Ab}$ positive patient subsequently developed LN compared with $1(1.85 \%)$ C1Q Ab negative patient $(p=0.173)$. There was no correlation between the level of C1Q Ab and the rate of $\mathrm{LN}$ flares.
Conclusion C1Q Ab has a known correlation with LN, however, its ability to predict flares has been less well characterized. Our prospective analysis shows that although the C1Q $\mathrm{Ab}$ positive patients were more likely to have a flare of $\mathrm{LN}$ in the following year, there was not a statistically significant difference between the $\mathrm{C} 1 \mathrm{Q} \mathrm{Ab}$ positive and negative groups. In addition, only a relatively small proportion of C1Q $\mathrm{Ab}$ positive patients went on to have a flare (20\%). Our data therefore does not support the use of $\mathrm{C} 1 \mathrm{Q} \mathrm{Ab}$ in predicting a flare of $\mathrm{LN}$.

\section{P14 MICROARRAY ANALYSIS IDENTIFIES ANTI-CPG ANTIBODIES TO BE STRONGLY ASSOCIATED WITH SLE AND LUPUS NEPHRITIS}

${ }^{1}$ Tammo Brunekreef, ${ }^{1}$ Maarten Limper, ${ }^{2}$ Linda Mathsson-Alm, ${ }^{2}$ Jorge Dias, ${ }^{1}$ Jaap van Laar, ${ }^{3}$ Imo Hoefer, ${ }^{3}$ Saskia Haitjema, ${ }^{4}$ Henny Otten. ${ }^{1}$ Dept. of Rheumatology and Clinical Immunology, UMC Utrecht, Utrecht, The Netherlands; 'Thermo Fisher Scientific, Uppsala, Sweden; ${ }^{3}$ Clinical Diagnostic Laboratory, UMC Utrecht, Utrecht; ${ }^{4}$ Center of Translational Immunology, UMC Utrecht, Utrecht, The Netherlands

\subsection{6/lupus-2020-eurolupus.63}

Background Many autoantibodies are known to be associated with SLE, although their role in clinical practice is limited because of low sensitivity and weak associations with clinical manifestations. Therefore, there has been great interest in the discovery of new autoantibodies or autoantibody patterns for clinical practice. In this study, we investigated patterns of new and known antibodies and their possible role in diagnostics or risk stratification.

Methods Between 2014 and 2017, residual sera of all antidsDNA tests in the UMC Utrecht were stored in a biobank. Diagnosis and presence of symptoms at each blood draw were retrospectively assessed in the patient records with the Utrecht Patient-Oriented Database (UPOD) using a newly developed text mining algorithm. Sera from a balanced cohort of patients with different diagnoses and patients without an assigned diagnosis were analyzed for the presence of 74 autoantibodies by a custom-made immunofluorescent microarray. Whenever possible, results were compared to corresponding historic in-house tests to assess quality. Differences in autoantibodies between patients with SLE and patients with a low suspicion of SLE were investigated by univariate and machine learning (XGBoost) analyses.

Results Autoantibody profiles of 484 patients with SLE were compared to 218 controls. Results from the microarray corresponded well with those from corresponding validated assays (AUC 0.726-0.902). Both univariate and machine learning analysis showed anti-dsDNA as most distinctive feature between both groups. Moreover, antibodies against Cytosine-phosphate-Guanine (anti-CpG) DNA motifs were found to be strongly associated with SLE $(\mathrm{p}<0.0001)$ and lupus nephritis $(\mathrm{N}=161, \mathrm{p}=0.0015)$. AntidsDNA and anti-CpG antibodies correlated moderately with each other.

Conclusions Anti-CpG antibodies are prevalent in patients with SLE and are associated with Lupus Nephritis independent of anti-dsDNA, suggesting an additive diagnostic value of antiCpG antibodies.

Acknowledgements This project was supported by Thermo Fisher Scientific. 